\section{Controversies in obstetric anaesthesia}

Kai G. Smedstad MD, FRCPC
Since the days of Queen Victoria, the very act of providing pain relief for childbirth has been controversial. Although nobody today questions the efficacy and validity of modern obstetric analgesia and anacsthesia, there are those who query the need for the routine use of epidural analgesia in normal obstetrics, and there are anaesthetists who would rather not be involved in obstetrics. The objections to chloroform and ether were based on religious rather than medical tenets. These days women object to technological intrusions in the birthing process, and practitioners worry about medical complications and legal repercussions should anything go wrong.

Modern obstetric anaesthesia is sophisticated. Our understanding of the pharmacological action of the drugs we use and the physiological changes that result when pain is relieved has increased in the last few years. This overview will try to examine some of the most common of these controversies.

\section{The test dose}

The use, efficacy and validity of the epidural test dose continues to be controversial. The ideal test dose should differentiate between correct placement of an epidural needle or catheter, and an intravascular or intrathecal placement or injection. Thus, the test dose needs to fulfil two separate criteria: (1) contain a marker to identify intravascular injection without causing systemic toxicity; (2) provide a reliable, easily detected and safe, sensory and/or motor block if injected into the subarachnoid space. There is no perfect agent for this purpose. The marker for intravascular injection is usually epinephrine. Moore' reported that the addition of $15 \mu \mathrm{g}$ of epinephrine to local anaesthetic solutions resulted in a 30 -beat increase in heart rate after 20 seconds when the drugs were injected intravenously in premedicated surgical patients. The validity of this study, when applied to women in labour, has been criticized by many authors, most notably Cartwright. ${ }^{2}$ During a contraction, the heart rate increases some 20-30 per cent, so the timing of the injection in relation to the contraction is, therefore, crucial. The heart rate must be monitored continuously by an ECG and closely observed to pick up a sustained increase unrelated to labour itself. Even so, Cartwright showed that, statistically, if one relied upon heart rate increase alone to determine intravascular placement of the epidural catheter, there would be a false positive test in 12 per cent of patients. If the catheter was removed in all these patients it would be replaced unnecessarily in 12 out of 13 cases. Quite apart from its unreliability, the use of epinephrine as a marker is controversial. Twenty micrograms of IV epinephrine has been shown to decrease uterine blood flow in experimental models. In healthy mothers and babies the addition of epinephrine to the local anaesthetic for epidural analgesia has not been shown to be detrimental, but doubt still remains whether the use of this drug is safe where placental blood flow is compromised, in pre-eclamptic patients, or in patients receiving $\beta$ adrenergic agonist tocolytic therapy. ${ }^{3}$ Other markers may prove to be better and safer. Baker et al. ${ }^{4}$ tested a number of chronotropic agents with local anaesthetics injected IV in the pregnant sheep model. They found that none of the solutions containing epinephrine $15 \mu \mathrm{g}$ was effective in increasing the maternal heart rate over 30 per cent, yet all were followed by a significant decrease in uterine blood flow and deceleration of the fetal heart rate, or diminished beat to beat variability. Isoproterenol, a pure $\beta$-agonist, 5 $\mu \mathrm{g}$ on its own or with lidocaine or bupivacaine effectively increased maternal heart rate without adverse effects on fetal heart rate or beat to beat variability. It may be that, in the future, better and safer test dose markers for intravascular placement will be used in obstetric practice.

Bupivacaine and lidocaine are the commonly used agents in obstetric anaesthesia. Chloroprocaine should not be injected into the cerebrospinal fluid. Bupivacaine does not give reliable indication of subarachnoid block quickly. Mitchell ${ }^{5}$ reported that $5 \mathrm{ml}$ of 0.5 per cent bupivacaine did not produce widespread and obvious spinal block by six minutes, and reliance on voluntary movements only would have failed to reveal subarachnoid injection since 95 per cent of his patients were able to flex their ankles at six minutes. A greater proportion of patients had motor block after hyperbaric bupivacaine was injected in the subarachnoid space, but again there was a delay up to 20 minutes before 90 per cent of patients were unable to flex their ankles. Sensory block, however, was demonstrated in the great majority of his patients at five minutes. Careful examination is, therefore, necessary to ascertain subarachnoid block using bupivacaine.

Department of Anaesthesia, McMaster University, Hamilton, Ontario. 
Two $\mathrm{ml} 1.5$ per cent lidocaine in 7.5 per cent dextrose resulted in sensory block of S-2 within two minutes in Abraham's patient, ${ }^{6}$ whereas the block was much slower in onset after correct epidural injection. Biehl ${ }^{7}$ uses $2 \mathrm{ml}$ two per cent lidocaine plain as a test dose for subarachnoid block, and checks both motor and sensory variables after five minutes. ${ }^{7}$ Crawford ${ }^{8}$ points out that none of these drugs will invariably afford evidence of inadvertent intravascular or subarachnoid injection, and Scott $^{9}$ concurs that "the most dangerous aspect of a false negative test dose is the confidence it inspires in the anaesthetist that all is well and the main dose can be injected with impunity."

A prudent precaution, even when using test doses, is to inject the anaesthetic drug slowly and in aliquots of $5 \mathrm{ml}$, wait one to two minutes and assess the patient's response by talking to her, asking about symptoms of toxicity.

\section{The use of epidural analgesia in controversial conditions}

\section{Bacterial infections}

Practitioners continue to ask whether to use epidurals for pain relief in febrile patients. Recent discussion sheds some light on this. ${ }^{10}$ In a study of febrile patients in labour, it was found that 90 per cent of those with fever did not have bacteraemia and in patients with chorioamnionitis only eight per cent were found to have bacteraemia. There is no demonstrable relationship between the patient's temperature and the probability of bacteraemia, and 49 per cent of patients with positive blood cultures have temperatures of less than $38.8^{\circ} \mathrm{C}$. In contrast, we may find transient bacteraemias in eight per cent of patients after bladder catheterization, even if their urine is sterile. It is very difficult to determine the risk of inserting epidural catheters in febrile parturients. These patients are often in preterm labour and may have prolonged and painful contractions necessitating instrumentation or pitocin augmentation. Given the low incidence of bacteraemia, and the lack of any studies or even case reports detailing the association between epidural abscess formation and use of epidural analgesia in febrile parturients, it seems unreasonable to deny such pain relief when indicated.

\section{Herpes simplex and zoster}

Recent evidence suggests that the use of spinal opioids (epidural morphine or fentanyl) for pain relief after Caesarean sections ${ }^{11}$ may reactivate herpes simplex Type I virus in the post-partum period. The reactivation seems to be associated with facial pruritus. In a prospective study, the overall incidence of post-partum HSVL lesions in all parturients was 2.6 per cent; 9 per cent of patients given epidural opioids compared with one per cent of patients not receiving this had reactivation of the virus. 6.8 per cent of patients developed HSVL after Caesarean sections, 13 out of the total number of 14 of these had received epidural morphine postoperatively. The addition of epinephrine to the epidural solution intensified the itching. The mechanism of reactivation is unknown, perhaps it is associated with endogenous opioid activity within the spinal nucleus of the trigeminal nerve. Reactivation of HSVL in the trigeminal nerve raises concern regarding this infection in the eye. There are no studies or reports of reactivation of genital herpes simplex HSVL Type II with epidural opioids. It has been reported, however, that herpes zoster virus can be activated after spinal anaesthesia. A recent retrospective study on anaesthesia for Caesarean section in patients with genital herpes infections ${ }^{12}$ concluded that patients with positive viral cultures in the last month of pregnancy, or who had active secondary lesions did not develop any complications from regional anaesthesia. The presence of primary HSVL Type II lesions may well be a contraindication to spinal or epidural anaesthesia, especially if the patient has generalized symptoms.

\section{AIDS}

There are no studies or reports on the use of epidural analgesia in labour for the patient who is HTLV III positive. Bear in mind that 20 per cent of patients with AIDS develop vacuolar degeneration of the spinal cord with paraplegia, ataxia and incontinence and 30 per cent develop subacute encephalitis. It is theoretically possible to activate latent virus in the central nervous system and the potential exists for serious flareups secondary to unknown factors in patients with inactive disease. Such patients should be informed of the potential risks before using epidural analgesia in labour. ${ }^{13}$

\section{Continuous infusion epidural analgesia}

Continuous infusion of local anaesthetics using mechanjcal infusion pumps has gained popularity in recent years. New pumps, specifically approved for this purpose are now on the market. The advantages of continuous infusion over traditional "top-ups" (or "catch-ups") of intermittent injections are more continuous analgesia, stable haemodynamic variables and lessened risks from inadvertent misplacement of catheters. ${ }^{14}$

Studies have confirmed that patient satisfaction is high with continuous infusion. ${ }^{15-18}$ All patients receiving continuous infusions first have a starting dose of local anaesthetic injected through the catheter. The response to this dose is then assessed while the anaesthetist is present and before the infusion is connected. There is general agreement in these studies that good sensory blockade is obtained and the block does not usually extend above T6. 
The incidence of missed segments is similar to that seen with intermittent "top-ups," and some patients on infusions may need additional doses of drugs by bolus injection to combat such pain.

Motor block varies between studies. We found no difference in the incidence of motor block using infusion or injection of 0.25 per cent bupivacaine $8 \mathrm{ml}^{~} \mathrm{hr}^{-1}$. With more dilute solutions, the incidence of motor block was less. ${ }^{18}$ We found a higher incidence of forceps deliveries in primiparous parturients receiving an infusion compared with intermittent top-ups when 0.25 per cent bupivacaine was used. With dilute solutions $(0.125$ per cent or less) this has not been found.

The efficacy of the block is related to the spread of the drug and the number of dermatomes blocked. Therefore, a more dilute solution given in a higher volume will give a better sensory block with less motor involvement than the same total dose given using a high concentration in a low volume. ${ }^{19}$ This has also been shown to be the case with top-ups. ${ }^{20}$ Recent papers show a tendency to lower concentrations, such as 0.0625 per cent, or 0.075 per cent bupivacaine. The addition of a small dose of fentanyl to the infusate, for instance $2 \mu \mathrm{g} \cdot \mathrm{ml}^{-1}$ of fentanyl in 0.0625 per cent bupivacaine ${ }^{21}$ improves pain relief with minimal motor block without shortening labour.

Whether continuous infusion epidural analgesia is safer than intermittent top-ups remains to be proven. Since epidural mishaps are exceedingly rare when due precautions are taken, a series of cases would be necessary to prove this point. However, rapid onset of the spinal block or toxicity from vascular injection continues to occur with bolus administration. Slow progression of spinal block to $\mathrm{T} 4$ from infusion of $5 \mathrm{ml} \cdot \mathrm{hr}^{-1} 0.025 \mathrm{per}$ cent bupivacaine has been described without any untoward effects to the patient, whereas symptoms of toxicity from continuous intravascular infusion have not been reported. ${ }^{22}$ It is important to stress that continuous infusion epidural analgesia should be conducted with the same vigilance and precautions as used with intermittent injections.

\section{Epidural opioids in obstetric analgesia}

As recently as 1983 , a review article concluded that "epidural narcotics have no clinical usefulness in obstetrics." Since then, much experience has been gained using opioids to augment epidural local anaesthetics for labour pain, or on their own for post-partum pain relief after operative delivery. The current "state of the art" indicates that there is a place for spinal opioids in the labour suite and the section room and beyond.

Over the last six years there have been many reports on the improved analgesia obtained by adding $50-100 \mu \mathrm{g}$ of fentanyl to bupivacaine. A comprehensive study by
Cohen ${ }^{23}$ found good pain relief when fentanyl was added to 0.2 per cent and 0.068 per cent bupivacaine in a volume of $11 \mathrm{ml}$, but the improvement when compared with the local anaesthetic alone did not reach statistical significance. However, when a subanalgesic concentration of bupivacaine is used, addition of fentanyl will improve the analgesic effect. The addition of fentanyl to epidural bupivacaine is not associated with an increase in maternal or fetal/neonatal side effects. Brownridge ${ }^{24}$ has had extensive experience with meperidine-bupivacaine mixtures for analgesia in obstetrics. He uses 0.125 per cent bupivacaine with 0.25 per cent meperidine in $10 \mathrm{ml}$ increments. His latest prospective study deals with patient satisfaction and acceptance of the technique. Disadvantages of epidural analgesia, such as leg weakness, numbness, and reduced awareness of the progress of birth, and a higher incidence of technical interventions, can be diminished by reducing the density of neural blockade, but the quality of analgesia may also become less predictable and reliable. In this study 100 mothers ( 57 primiparas, 48 per cent induced labour) requesting epidural analgesia were questioned about their pain relief after receiving the bupivacaine-meperidine mixture in an initial dose of $10 \mathrm{ml}$ and "top-ups" of $10 \mathrm{ml}$ on request. Mothers who wished to sit in a chair or walk around were encouraged to do so. Ninety per cent described pain relief as adequate or satisfactory. Rectal or perineal pain is often not blocked adequately with low-dose epidural mixtures, yet overall satisfaction with this form of pain relief seems related to the mother's ability to move and be in control throughout her labour, and the lack of side-effects. A low dose bupivacaine-opioid mixture is inadequate for instrumental delivery and episiotomy, and "top-up" and/or local infiltration with stronger local anaesthetic solutions are recommended for such patients.

A recent study from France compared continuous infusion versus repeated injections of bupivacainefentanyl during labour. ${ }^{25}$ Although both forms of epidural administration provided excellent pain relief, the workers demonstrated a clear advantage of continuous infusion techniques in terms of the quality of analgesia obtained. Increasing the fentanyl dosage does not improve analgesia. Drug accumulation or tachyphylaxis has not been reported when continuous infusion techniques are employed.

In another recent study ${ }^{21}$ Chestnut et al. reported good to excellent analgesia with 0.0625 per cent bupivacaine with fentanyl, similar to that obtained with 0.125 per cent bupivacaine plain. Women in the bupivacainefentanyl group had less motor block, but there was no difference in duration of labour or frequency of spontaneous delivery.

Side effects are minimal with epidural fentanyl. Most 
authors report transient mild pruritus only. There is rapid onset of analgesia due to the spinal action of the opioid, with low and rapidly declining opioid blood levels, insufficient in itself to contribute to analgesia. ${ }^{26}$ Advantages of using lipid soluble opioids mixed with low concentration local anaesthetics in labour appears to be good analgesia with less local anaesthesia, less motor block, a high degree of patient satisfaction and minimal side effects.

Extradural and intrathecal opioids are also used for relief of pain during and after Caesarean section. ${ }^{27}$ Morphine, due to its lower lipid solubility, has a longer latency before analgesia is achieved, but a longer duration of action (12-24 hours). Undesirable side effects: pruritus, vomiting and urinary retention occur, and delayed respiratory depression has been reported, albeit rare.

Fentanyl and meperidine are more lipid soluble, have a shorter onset time, and a shorter duration of action (three to six hours), but the incidence of side effects is much less and delayed respiratory depression has not been described with fentanyl. Fifty $\mu \mathrm{g}$ of epidural fentanyl will quickly alleviate discomfort experienced during intraabdominal manipulation at Caesarean section; $100 \mu \mathrm{g}$ will help alleviate postoperative pain and decrease narcotic requirements significantly in the first 24 hours.

Further clinical experience with these drug mixtures should help settle existing controversies, and answer questions regarding improved efficacy and safety.

\section{Epidurals and coagulopathy}

Conventional wisdom dictates that anaesthetists do not perform spinal or epidural blocks in patients who are anticoagulated or have coagulopathies. An epidural haematoma is a true emergency requiring prompt surgical decompression to avoid permanent cord injury. Yet reports of such emergencies are few. Some have been spontaneous occurrences, some associated with lumbar puncture in fully anticoagulated patients. ${ }^{28}$ Epidurals are used most commonly in Canada in pregnant patients for obstetric anaesthesia and analgesia - yet there are no reports of an epidural haematoma in this population in our country.

Normal women in labour may have platelet counts that vary widely. Rolbin et al. ${ }^{29}$ recently reported a mean platelet count of $275 \pm 86 \times 10^{9} \cdot \mathrm{L}^{-1}$ in pregnancy. Unexplained periparturient thrombocytopaenia: a platelet count below $150 \times 10^{9} \cdot \mathrm{L}^{-1}$ occurred in 6.4 per cent of the women. Sixty-one of these 104 women requested epidural analgesia and received it either by continuous or single injection method. In most of these patients, as is usually the case when normal women request an epidural, the platelet count was not available when regional analgesia was instituted. The platelet count usually returns to normal within a week of delivery where there is no underlying pathology. No complications from insertion of epidural needles have been reported in such instances, and it is reasonable to assume that many women with platelet counts below $150 \times 10^{9} \cdot \mathrm{L}^{-1}$ have received epidural anaesthesia over the years. In women with no underlying pathology such as toxaemia of pregnancy, immune thrombocytopaenic purpura, collagen disorders or other bleeding diatheses, one can safely use epidural analgesia if the platelet count is over $100 \times$ $10^{9} \cdot \mathrm{L}^{-1}$

The situation is different in toxaemia. Thrombocytopaenia is the most common haemostatic abnormality of preeclampsia, and is caused by increased platelet destruction. Thrombocytopaenia occurs in 15 per cent of preeclamptic patients, and it may be the earliest abnormality detected. The mechanism for the destruction of platelets in preeclampsia is not clear. Burrows et al ${ }^{30}$ suggest that an immune mechanism may be responsible as demonstrated by elevated levels of platelet-associated IgG in 45 per cent of thrombocytopaenic preeclamptic patients.

Patients who are not preeclamptic may have normal bleeding time when the platelet count is over $50,000 \cdot \mathrm{L}^{-1}$. however, in preeclamptic patients the bleeding time is often severely prolonged, even with an acceptable platelet count, and should be carried out before inserting an epidural if platelet function is in doubt. ${ }^{31}$

Lumbar epidural anaesthesia is the method of choice for Caesarean section in preeclampsia provided the platelet count is over 100,000 and the coagulation status is otherwise normal. ${ }^{32}$ In severe preeclampsia platelet counts may change rapidly, as may other indicators of coagulation status. It is important, therefore, to obtain serial counts and use clinical judgement before instituting regional anaesthesia. Transfusion of platelets and other coagulation factors may be necessary before and during operative delivery, and it is of great help to consult haematology when dealing with these cases.

\section{Conclusion}

Lumbar epidural analgesia remains the best method of relieving labour pain and it can be quickly converted to anaesthesia for operative delivery if the need arises.

Epidural analgesia is safe when care is taken to prevent complications.

All health care professionals looking after women in labour should know about the potential problems associated with invasive analgesic methods and be trained to recognize and deal with these, preferably before anything serious happens. It is our responsibility as anaesthetists to provide this training. It is also our responsibility to inform our patients of the advantages as well as the potential hazards of obstetric analgesia in such a way that we do not 
alarm them unneccessarily, but give them a truly informed choice. This is best done before the woman is in labour; an information pamphlet is often used for this purpose. Obstetric anaesthesia is an exciting and evolving specialty. I hope to see the anaesthetist truly becoming a team member in obstetric care in all our hospitals.

\section{References}

1 Moore DG, Batra SM. The components of an effective test dose prior to epidural block. Anesthesiology 1981; 55: 693-6.

2 Cartwright $P D, M c$ Carroll SM, Antzaka C. Maternal heart rate changes with a plain epidural test dose. Anesthesiology 1986; 65: 226-8.

3 Dain SL, Rolbin SH, Hew EM. The epidural test dose in obstetrics: is it necessary? Can J Anaesth 1987; 34: 601-5.

4 Baker BW, Longante S, Jones MM et al. The epidural test dose in obstetrics reconsidered. SOAP Abstracts 1987; 19: 69.

5 Mitchell RW, Dowler GM, Scott DB. Effects of posture and baricity on spinal anaesthesia with 0.5 per cent bupivacaine $5 \mathrm{ml}$. A double-blind study. Br J Anaesth 1988; 61: 139-43.

6 Abraham RA, Harris AP, Maxwell LG, Kaplow S. The efficacy of $1.5 \%$ lidocaine with $7.5 \%$ dextrose and epinephrine as an epidural test dose for obstetrics. Anesthesiology 1986; 64: 116-9.

7 Biehl DR. The dilemma of the epidural test dose. Can J Anaesth 1987; 34: 545-8.

8 Crawford JS. Epidural test dose in obstetrics. Can J Anaesth 1988; 35: 441-2.

9 Scott DB. Test doses in extradural block. BR J Anaesth 1988; 61: 129-30.

10 Davies $J M$, Thistlewood JM, Rolbin SH, Douglas MJ. Infections and the parturient: anaesthetic considerations. Can J Anaesth 1988; 35: 270-7.

11 Coone LAL, Conley JM, Clark KM et al. Recurrent herpes simplex virus labialis and the use of epidural morphine in obstetric patients. Anesth Analg 1988; 67: 318-23.

12 Ramanathan $S$, Sheth $R$, Turndorf $H$. Anesthesia for cesarean section in patients with genital herpes infections: a retrospective study. Anesthesiology 1986; 64: 807-9.

13 Greene CR Jr. Spinal and epidural anesthesia in patients with the acquired immunodeficiency syndrome. Anesth Analg 1986; 65: 1090-1.

14 Continuous extradural analgesia: catch-up or top-up? Lancet 1987; 1: 1300-1.

15 Smedstad KG, Morison DH. A comparative study of continuous and intermittent epidural analgesia for labour and delivery. Can J Anaesth 1988; 35: 234-41.
16 Hicks JA, Jenkins JO, Newton MC, Findley $I L$. Continuous epidural infusion of 0.075 per cent bupivacaine for pain relief in labour. A comparison with intermittent top-ups of 0.5 per cent bupivacaine. Anaesthesia 1988; 43: 289-92.

17 Li DF, Rees $G A D$, Rosen $M$. Continuous extradural infusion of $0.0625 \%$ of $0.125 \%$ bupivacaine for pain relief in primigravid labour. Br J Anaesth 1985; 57: 264-70.

18 Abboud TK, Afrasiabi A, Sarkis $F$ et al. Continuous infusion epidural analgesia in parturients receiving bupivacaine, chloroprocaine, or lidocaine - matemal, fetal, and neonatal effects. Anesth Analg 1984; 63: 421-8.

19 Bogod DG, Rosen $M$, Rees GA. Extradural infusion of $0.125 \%$ bupivacaine at $10 \mathrm{ml} \cdot \mathrm{h}^{-1}$ to women during labour. Br J Anaesth 1987; 59: 325-30.

20 Purdy G, Currie J, Owen $H$. Continuous extradural analgesia in labour. Comparison between "on demand: and regular top-up injections." Br J Anaesth 1987; 59: 319-24.

21 Chestmut DH, Owen CL, Bates JN, Ostman LG, Choi WW, Geiger $M W$. Continuous infusion epidural analgesia during labour: a randomized, double-blind comparison of $0.0625 \%$ bupivacaine $/ 0.0002 \%$ fentanyl versus $0.125 \%$ bupivacaine. Anesthesiology 1988; 68: 754-9.

22 Matouskova A. Epidural analgesia: continuous miniinfusion of bupivacaine into the epidural space during labor. Acta Obstet Gynecol Scan 1979; Suppl 83: 5-52

23 Cohen SE, Tan S, Albright GA, Halpern J. Epidural fentanyl/bupivacaine mixtures for obstetric analgesia. Anesthesiology 1987; 67: 403-7.

24 Brownridge $P$. Epidural bupivacaine-pethidine mixture clinical experience using a low-dose combination in labour. Aust NZ J Obstet Gynaecol 1988; 28: 17-24.

$25 D^{\prime}$ Athis $F$, Macheboeuf M, Thomas $H$ et al. Epidural analgesia with a bupivacaine-fentanyl mixture in obstetrics: comparison of repeated injections and continuous infusion. Can J Anaesth 1988; 35: 116-22.

26 Cousins MJ. Comparative pharmacokinetics of spinal opioids in humans: a step toward determination of relative safety. Anesthesiology 1987; 67: 875-6.

27 Nauly JS, Datta S, Ostheimer GW, Johnson MD, Burger $G A$. Epidural fentanyl for postcesarean delivery pain management. Anesthesiology 1985; 63: 694-8.

28 Owens $L$, Kastin $G$. Spinal subarachnoid haematoma after lumbar puncture and heparinization. Anesth Analg 1986; 65: 1201-7.

29 Rolbin SH, Abbott D, Musclow E, Papsin F, Lie LM, Freedman $J$. Epidural anesthesia in pregnant patients with low platelet counts. Obstet Gynecol 1988; 71 : 918-20.

30 Burrows RF, Hunter DJS, Andrew M, Kelton JG. A prospective study investigating the mechanism of 
thrombocytopenia in preeclampsia. Obstet Gynecol 1987; 70: 334-8.

31 Kelton JG, Hunter DJS, Neame PB. A platelet function defect in preeclampsia, Obstet Gynecol 1985; 65:107-9.

32 Morison DH. Continuing Medical Education Article: Anaesthesia and pre-eclampsia. Can J Anaesth 1987; 34: 415-22. 